\title{
Role of pegaptanib sodium in the treatment of neovascular age-related macular degeneration
}

\author{
Sobha Sivaprasad \\ Laser and Retinal Research Unit, \\ King's College Hospital, UK
}

Correspondence: Sobha Sivaprasad King's College Hospital, Denmark Hill, London, SE5 9RS, UK

Tel +442032994548

Fax +44 2032993738

Email senswathi@aol.com

\begin{abstract}
Age-related macular degeneration (AMD) is responsible for more than half the blind registration in the United Kingdom. Retinal manifestations of AMD can be categorized as either atrophic or neovascular. The hallmark of AMD is the development of choroidal neovascularization $(\mathrm{CNV})$. Until recently, there have been few, limited treatment modalities (eg, photodynamic therapy [PDT]) for this condition and the mainstay of treatment has comprised social and lifestyle support. However, increased understanding of the molecular processes at work in neovascular AMD and CNV in recent years has led to the introduction of new antiangiogenic agents that target vascular endothelial growth factor (VEGF). These agents either inhibit a selected VEGF isoform (eg, VEGF ${ }_{165}$ inhibition by pegaptanib sodium) or inhibit all forms of the VEGF isoform (eg, non-selective VEGF blockade by ranibizumab). The trial data suggest that non-selective inhibition of VEGF offers better treatment outcomes in neovascular AMD. As a result, agents that inhibit all VEGF isoforms are now widely used as first-line therapy for this condition. However, it is known that VEGF plays an important role in maintaining the intergrity of the cardiovascular system and, particularly as the age of patients with AMD places them at an elevated risk of thromboembolic events, long-term post-marketing surveillance data are essential to determining whether non-selective VEGF blockade confers any increased risk. Theoretically, selective VEGF inhibition may reduce any risk associated with pan-VEGF blockade, yet on the basis of initial trials, their use remains more limited at this time. However, clinical practice suggests that initial trials may have under-estimated the efficacy of selective-VEGF inhibition. Observational studies also indicate that better treatment outcomes may be possible by combining VEGF inhibitors sequentially with each other, or with existing therapies (eg, photodynamic therapy [PDT]). The optimum role and indications of anti-VEGF agents will come through careful consideration of the available efficacy and safety data, from the outcomes of long-term follow-up studies, and through assessment of the relative merits of the two approaches to VEGF inhibition in clinical practice. At this time, further head-to-head trials, and economic evaluations, comparing the treatment alternatives are needed.
\end{abstract}

Keywords: age-related macular degeneration (AMD), choroidal neovascularization (CNV), vascular endothelial growth factor (VEGF), cardiovascular, ranibizumab, pegaptanib sodium

\section{The disease burden}

Age-related macular degeneration (AMD) is the leading cause of blindness in the western world's elderly population (Klein et al 1992). The early form of the disease is characterized by the presence of drusen in the central macula and/or retinal pigment epithelial (RPE) changes; it is often referred to as age-related maculopathy (ARM). More advanced AMD - which can take either a neovascular/exudative/wet form characterized by choroidal neovascularization (CNV), or a dry form - can lead to severe visual loss.

Dry AMD is associated with a very gradual loss of vision and is responsible for $20 \%$ of the legal blindness owing to AMD. Neovascular AMD is responsible for the other $80 \%$ and has a more variable course. Disease progression tends to be quicker 
with neovascular AMD and visual acuity (VA) can change from normal vision to legal blindness within a matter of weeks (Ferris et al 1984).

Worldwide, there are approximately 500,000 new cases of neovascular AMD diagnosed annually (Bressler et al 2003). In the United Kingdom (UK), there is an estimated 245,000 people living with neovascular AMD, and it represents more than 50\% of the country's blind registration (Evans 1995; Owen et al 2003). This huge public health impact promises to exact an even greater toll with the imminent demographic right-shift seen in increasingly aging populations.

\section{Disease characteristics}

The hallmark of neovascular AMD is the development of CNV. Patients may present with several pathological features, such as sub-retinal fluid (SRF), hemorrhages and exudates, pigment epithelial detachments (PED), and retinal scarring. The CNV can develop directly under the center of the fovea (subfoveal CNV), in the remainder of the fovea (juxtafoveal CNV), or in the area of the macula excluding the fovea (extrafoveal CNV). Angiographically, a neovascular lesion is classified depending on the amount of classic CNV present: lesions with $\geq 50 \%$ classic CNV are termed predominantly classic lesion, while lesions with $<50 \%$ classic $\mathrm{CNV}$ are defined as minimally classic lesions. Lesions with no classic CNV are termed occult lesions. Those lesions with classic neovascular membranes are associated with faster progression to legal blindness than occult neovascular membranes (Bressler et al 2002).

\section{Disease management}

The mainstay of existing treatment for dry AMD and for the advanced neovascular form of the condition comprises social support, visual rehabilitation, and provision of low vision aids. The traditional treatment modalities for wet AMD are few and, at best, halt disease progression (Macular Photocoagulation Study Group 1993; Treatment of Age-Related Macular Degeneration with Photodynamic Therapy [TAP] Study Group 1999).

\section{Laser photocoagulation}

Laser photocoagulation was the first evidence-based treatment option found to be beneficial in certain subtypes of neovascular membranes. It is possible to treat well-defined, classic, extrafoveal lesions with an argon, krypton, or diode laser. The aim of laser treatment is to halt the rapid visual loss caused by progression of CNV within the retinal membranes. However, treatment is limited and is associated with high recurrence rates (up to 50\%), limited lesion eligibility for treatment (only 10\%-15\%), and an immediate drop in vision when used for the treatment of subfoveal CNV (Macular Photocoagulation Study Group 1993).

\section{Photodynamic therapy}

In 2000, the Food and Drug Administration (FDA) and European Agency for the Evaluation of Medicinal Products (EMEA) approved verteporfin (Visudyne ${ }^{\circledR}$; QLT Therapeutics, Inc, Vancouver, British Columbia, Canada, and Novartis Ophthalmics, Bulach, Switzerland) for use in patients with predominantly classic, subfoveal CNV secondary to AMD. In the UK, the National Institute for Health and Clinical Excellence (NICE) has also endorsed the use of photodynamic therapy (PDT) with verteporfin for the treatment of subfoveal, predominantly classic lesions (with no occult subfoveal CNV) (NICE 2003). PDT has two components; injection of a photosensitive agent and subsequent application of a low-energy laser to the affected areas of the retina. Akin to laser photocoagulation, the approach aims to prevent further loss of vision by halting progression in the neovascular membranes. Both treatment modalities are destructive procedures (Bressler and Bressler 2000), but a new treatment avenue has been opened through recent research that has led to the development of targeted therapies for AMD.

\section{Targeted therapies}

Understanding of the molecular processes at work in AMD and CNV has increased significantly in the last decade. Several lines of evidence indicate a multi-factorial etiology, one that involves a combination of genetic factors, inflammation, accumulation of lipid and metabolic by-products, oxidative stress and alterations in the Bruch's membrane (Ambati et al 2003). Responding to these stresses, the RPE and retina produce various growth factors such as vascular endothelial growth factor (VEGF), fibroblast growth factor-2, and pigment epithelium-derived growth factor (Dorrell et al 2007). Although VEGF is integral to the physiological processes that require angiogenesis (such as embryogenesis, somatic growth and tissue repair) (Stephan and Brock 1996), it is also implicated as central to the pathological processes involving neovascularization, such as the development of CNV (Lopez et al 1996; Bhisitkul 2006).

\section{Rationale for targeting VEGF in neovascular AMD}

VEGF acts as an endothelial-cell chemo-attractant and antiapoptotic agent, leading to endothelial-cell proliferation, 
survival, and migration, as well as to increased vascular hyperpermeability. It induces the expression of urokinase- and tissue-type plasminogen activators, as well as metalloproteinase interstitial collagenase (Ferrara 2004). This co-induction promotes degradation of the local extracellular matrix and facilitates endothelial cell migration ( $\mathrm{Ng}$ and Adamis 2005).

VEGF is a member of the platelet-derived growth factor family, and its production is often stimulated by tissue hypoxia or other growth factors (Pertovaara 1994). The VEGF gene gives rise to various polypeptides, including the 121-, 165-, 189-, and 208-amino acid isoforms ( $\mathrm{Ng}$ and Adamis 2005). Each VEGF isoform has unique characteristics, and $\mathrm{VEGF}_{165}$ is the form most abundant in the human eye, although it is ubiquitously distributed (Houck et al 1991). VEGF binds to two receptors, VEGFR-1 and VEGFR-2, and this binding activates signal transduction cascades (Usui et al 2004).

Several studies have investigated the role of VEGF in ocular neovascularization (Aiello et al 1994; Pierce et al 1996), and VEGF has been implicated in the development of retinal and iris neovascularization in ischemic diseases such as diabetic retinopathy (Adamis et al 2006), retinal vein occlusion (Pe'er et al 1998), retinopathy of prematurity (Chen and Smith 2007), and neovascular glaucoma (Tripathi et al 1998). In addition, evidence has also emerged to suggest a putative role for VEGF in CNV. VEGF levels have been found to be increased in the RPE and choroidal blood vessels of maculae exhibiting AMD (Frank et al 1996). The growth factor induces proliferation of choroid endothelial cells in non-human primates (Tolentino et al 1996), and adenoviral transfection of the VEGF gene into the RPE of rats has been shown to lead to the development of $\mathrm{CNV}$ (Baffi et al 2000).

Inhibition of VEGF can also impede the formation of CNV in experimental models: intravitreous (ITV) injection of an oligonucleotide targeted to the VEGF sequence has been shown to inhibit laser-induced $\mathrm{CNV}$ in rats (Eyetech Study Group 2002). In addition, repeated ITV injections of rhuFabV2, an active fragment of a recombinant humanized monoclonal VEGF antibody, inhibits the development of laser-induced CNV in a primate model (Eyetech Study Group 2002). These observations, which provide a sound rationale for targeting VEGF in an attempt to impede the progress of $\mathrm{CNV}$, combined with recent advances in directed molecular interventions (eg, monoclonal antibodies, aptamers, ribozymes, gene transfer therapy) have paved the path to the development of several anti-VEGF agents in the treatment of neovascular AMD (Dorrell et al 2007).

\section{Selective VEGF inhibition}

Pegaptanib sodium(Macugen ${ }^{\circledR}$, [OSI] Eyetech Pharmaceuticals, Pfizer) is the first targeted anti-VEGF therapy available for the treatment of CNV (Krzystolik et al 2002). The agent belongs to a new class of therapeutic drugs called aptamers. Aptamers are synthetic oligonucleotides that adopt a specific three-dimensional conformation that allows them to bind, with high specificity and affinity, to a wide range of therapeutic agents. These compounds are chemically synthesized from component nucleotide bases with reverse transcription and polymerase chain reaction technologies (Gragoudas et al 2004). Pegaptanib sodium is a 28-base ribonucleic acid (RNA) oligonucleotide with two branched $20 \mathrm{kDa}$ polyethylene glycol (PEG) moieties attached in order to increase the half-life of the drug in the vitreous cavity. The RNA sugar background is modified to prevent its degradation by endogenous endoand exo-nucleases. Pegaptanib sodium specifically targets the VEGF $_{165}$ isoform (Waheed et al 2004).

Phase I and II studies have evaluated the safety and efficacy of pegaptanib sodium as a therapy for subfoveal CNV secondary to AMD and have demonstrated that, given every 6 weeks, pegaptanib sodium provides anatomic and visual benefits (Ruckman et al 1998).

\section{The VISION study}

The VEGF Inhibition Study in Ocular Neovascularisation (VISION) consists of two concurrent, prospective, doseranging, multi-center (involving 117 centers and 1208 patients), randomized controlled trials (RCTs) that were conducted to assess the safety and efficacy of pegaptanib sodium in patients with CNV secondary to AMD (Gonzales and VISION Clinical Trial Group 2005). The VISION study population was broad, including patients aged $\geq 50$ years with any angiographic subtype of subfoveal CNV in the study eye. Any lesion $\leq 12$ disc areas, with scarring $>25 \%$ and hemorrhage $>50 \%$ of the total lesion size, was eligible for inclusion. The best corrected VA of the study eye ranged between $20 / 40$ and 20/320. Patients were randomly assigned either to sham injection or ITV injection of $0.3 \mathrm{mg}, 1 \mathrm{mg}$ or $3 \mathrm{mg}$ pegaptanib sodium. Injections were administered every 6 weeks for a total of 48 weeks (equating to nine separate injections). Patients receiving sham or study medications were treated identically except for the absence of scleral penetration in the sham patient group. All treatment arms allowed treatment with PDT with verteporfin (Visudyne) for predominantly classic CNV to ensure that the sham group was equivalent to usual care treatment for neovascular AMD.

The primary study outcome measure was the proportion of patients who lost $<15$ letters of VA (defined as 
3 lines on the study eye chart) at the end of week 54 . Additional efficacy end-points included: proportion of patients maintaining or gaining $\geq 0,5,10$, or 15 letters, or losing $\geq 30$ letters; mean changes in VA from baseline to week 54, at 6-weekly intervals, and the proportion of patients with VA of 20/200 or worse in the study eye at week 54.

Of the 1208 patients involved in the study, 296 patients were randomized to receive sham injections; 294 to receive $0.3 \mathrm{mg}$ pegatanib sodium ITV; 300 to receive $1 \mathrm{mg}$ pegaptanib sodium ITV; and 296 to receive $3 \mathrm{mg}$ pegaptanib sodium ITV. Overall, the $0.3 \mathrm{mg}$ dose was found to be most effective, regardless of angiographic sub-type or lesion size. A comparison of the outcomes recorded in the $0.3 \mathrm{mg}$ pegaptanib sodium and sham study arms is summarized in Table 1.

The average number of PDT treatments required per patient post baseline was lower in the $0.3 \mathrm{mg}$ pegaptanib sodium study arm (1.44) than in the sham study arm (2.33). The 2-year data show that patients who continued with pegaptanib sodium treatment for 2 years experienced less vision loss than those patients who discontinued treatment (see Table 2). Analysis of the data also indicated that there was no evidence that any angiographic subtype of the lesion, level of VA at baseline, or lesion size precluded a treatment benefit.

\section{Safety}

Adverse events, such as vitreous floaters and inflammation, were more frequently noted among patients receiving pegaptanib sodium than those administered sham injections.

Table I Comparison of outcomes in the $0.3 \mathrm{mg}$ intravitreous $\underline{\text { pegaptanib sodium and sham injection patient groups at week } 54}$

\begin{tabular}{lllr}
\hline & $\begin{array}{l}\text { Pegaptanib } \\
\mathbf{( 0 . 3} \mathbf{~} \mathbf{~ g}) \\
\mathbf{n}=\mathbf{2 9 4}\end{array}$ & $\begin{array}{l}\text { Sham } \\
\mathbf{n}=\mathbf{2 9 6}\end{array}$ & P-value \\
& $70 \%$ & $55 \%$ & $<0.00$ I \\
\hline $\begin{array}{l}\text { Proportion that lost } \\
\text { fewer than I5 letters }\end{array}$ & 7.93 & 15.05 & $<0.050$ \\
$\begin{array}{l}\text { Mean visual loss (letters) } \\
\text { Proportion that maintained } \\
\text { or gained vision }\end{array}$ & $33 \%$ & $23 \%$ & 0.003 \\
$\begin{array}{l}\text { Proportion that gained } \\
5 \text { letters }\end{array}$ & $22 \%$ & $12 \%$ & $<0.050$ \\
$\begin{array}{l}\text { Proportion that gained } \\
\text { I0 letters }\end{array}$ & $11 \%$ & $6 \%$ & $<0.050$ \\
$\begin{array}{l}\text { Proportion that gained } \\
\text { I5 letters }\end{array}$ & $6 \%$ & $2 \%$ & $<0.050$ \\
$\begin{array}{l}\text { Proportion with severe } \\
\text { visual loss }\end{array}$ & $10 \%$ & $22 \%$ & $<0.00$ I \\
$\begin{array}{l}\text { Proportion with vision } \\
\leq 20 / 200\end{array}$ & $38 \%$ & $56 \%$ & $<0.00$ I \\
\hline
\end{tabular}

Table 2 Impact of treatment discontinuation on visual outcome at week 102

\begin{tabular}{lll}
\hline & $\begin{array}{l}\text { Continued pegaptanib } \\
\text { sodium }(\mathbf{n}=\mathbf{I 3 3})\end{array}$ & $\begin{array}{l}\text { Discontinued } \\
\text { pegaptanib } \\
\text { sodium }(\mathbf{n}=\mathbf{1 3 2})\end{array}$ \\
\hline Mean visual loss & 0.6 letters & 5.3 letters \\
Loss of $\geq 15$ letters & $15.8 \%$ & $26.5 \%$ \\
Loss of $\geq 30$ letters & $2.3 \%$ & $4.7 \%$ \\
\hline
\end{tabular}

Serious adverse events with pegaptanib sodium included endophthalmitis (16/11636 injections), retinal detachment (13/11636 injections), and iatrogenic traumatic cataract (6/11636 injections). No increased risk of systemic adverse events was noted, but patients with high risk of cardiovascular and cerebrovascular events were excluded from the clinical trials.

\section{Role of pegaptanib sodium}

Pegaptanib sodium was the first anti-VEGF licensed for the treatment of $\mathrm{CNV}$ due to neovascular AMD. As understanding of the role of VEGF in the pathogenesis of CNV increases, many more trials are now turning their focus to the VEGF molecule. The benefit of pegaptanib sodium is that its efficacy is not dependent on lesion subtypes, lesion size, or baseline VA, allowing more patients to be eligible for treatment.

It is an aptamer and binds to VEGF with high specificity and affinity. It targets only $\mathrm{VEGF}_{165}$ and, as a result, is assumed not to interfere with other physiological actions of other isoforms of the molecule. Trial data demonstrate that the treatment effect occurs early and is sustained for 2 years, although the efficacy in the second year appears to decrease. The drug was found to be relatively safe over 2 years, and most adverse events reported in the study eyes were attributed to the injection procedure. The cumulative risk of endophthalmitis was found to be low in these trials.

\section{Non-selective VEGF inhibition Ranibizumab}

Other anti-VEGF agents are now being used in the treatment of neovascular AMD. Ranibizumab (Lucentis ${ }^{\circledR}$, Genentech, Inc) is an antibody binding site fragment with a molecular weight of $48 \mathrm{kDa}$ that is derived from a murine anti-VEGF antibody and is produced in an Escherichia coli expression system that is not glycosylated. It is a non-selective inhibitor of VEGF-A (Eyetech Study Group 2003). Data from two pivotal Phase III clinical trials on ranibizumab (Minimally classic/occult trial of the Anti-VEGF antibody Ranibizumab In the treatment of 
Neovascular AMD [MARINA] and Anti-VEGF Antibody for the Treatment of Predominantly Classic Choroidal Neovascularization in AMD [ANCHOR]) demonstrate that nearly all patients (approximately 95\%) treated with ranibizumab $(0.5 \mathrm{mg})$ maintained vision, and $\leq 40 \%$ reported vision improvement, at 1 year (Gaudreault et al 2005; Brown et al 2006; Rosenfeld et al 2006; Campochiaro 2007).

Patients treated with ranibizumab for minimally classic and occult lesions in the MARINA study experienced an improvement from baseline of 6.6 letters at 2 years compared with a loss of 14.9 letters in the sham study arm (Rosenfeld et al 2006). In the ANCHOR study, patients treated with ranibizumab for predominantly classic lesions experienced (on average) an 11.3 letter gain from baseline at 1 year compared with a loss of 9.5 letters in the PDT control group. Moreover, $40 \%$ of patients treated with ranibizumab achieved vision of $20 / 40$ or better.

\section{Bevacizumab}

Bevacizumab (Avastin ${ }^{\circledR}$, Genentech, Inc) is a full-length humanized monoclonal antibody to VEGF. It is produced in a Chinese hamster ovary mammalian cell system that is glycosylated, and it is designed and approved for intravenous administration for the treatment of metastatic colonic cancer. Similar to ranibizumab, it blocks all isoforms of VEGF-A, but importantly, the active binding sites of bevacizumab differ from those of ranibizumab. It has a molecular weight of $149 \mathrm{kDa}$ and has 100 times less binding affinity than ranibizumab (Steinbrook 2006).

Despite the differences between these two pan-VEGF inhibitors, it is well-hypothesized that bevacizumab is as effective as ranibizumab. Recently, several clinical series using ITV bevacizumab for the treatment of CNV secondary to AMD have shown promising results, reporting considerable improvement in VA, a reduction in retinal thickening (as detected from optical coherent tomography [OCT]) and no marked short-term toxicity (Cleary et al 2007; Emerson et al 2007; Wu et al 2008). However, due to its substantially higher molecular weight, local and systemic clearance of bevacizumab may be delayed, which could allow extended durability of effect of treatment and may result in higher systemic toxicity than ranibizumab.

\section{VEGF inhibition and thromboembolic risk}

The difference between these two approaches to VEGF inhibition is that pegaptanib sodium is a selective inhibitor of VEGF-A 165 isoform, while ranibizumab and bevacizumab are pan-VEGF-A blockers. The doses of the three agents used are small, but the plasma half-lives of both pegaptanib sodium (Macugen AMD Study group 2007) and bevacizumab are long compared with that of ranibizumab (Bakri et al 2007). Despite this, the peak plasma concentrations of pegaptanib sodium and ranibizumab are below $11-27 \mathrm{ng} / \mathrm{mL}$, the level necessary to inhibit the biologic activity of VEGF-A by half. However, the peak plasma concentration recorded for bevacizumab is substantially higher (Wong et al 2007).

The physiological level of serum VEGF is $<100 \mathrm{pg} / \mathrm{mL}$ (Larsson et al 2002). Physiologically, VEGF is required for both the integrity of the cardiovascular system and, pathologically, for re-perfusion of ischemic tissues (eg, the myocardium following myocardial infarction [MI] and the brain following stroke) (Zlokovic 2006; Infanger et al 2007). The supposition is, therefore, that chronic pan-VEGF blockade may increase cardiovascular risk. A recent report also suggested that the overall rates of arterial thromboembolic events recorded in MARINA were lower than those noted in population-based studies, suggesting selection bias due to under-recruitment of patients with existing cardiovascular disease (Liew et al 2007).

Wong et al suggest that AMD may be a marker of high cardiovascular risk (Wong et al 2007). Furthermore, analysis of safety outcomes in trials of ranibizumab reports a significant increase in non-ocular hemorrhage in the treatment arms (Liew et al 2007). Similarly, a recent interim analysis of the safety data on ranibizumab for the treatment of neovascular AMD (the on-going Safety Assessment of Intravitreal Lucentis fOR AMD [SAILOR] study) reported a higher rate of stroke in the patients with a prior history of stroke randomized to $0.5 \mathrm{mg}$ ranibizumab (9.6\%) compared with the rate recorded in those randomized to receive $0.3 \mathrm{mg}$ ranbizumab $(2.7 \%$ ) (Genentech 2007). However, this trend is inconclusive and the analysis did not find any difference in rate of MI or cardiovascular deaths between the two treatment groups. These data highlight the need for, and importance of, carrying out thorough assessment of long-term, post-marketing surveillance data regarding these agents. Clinicians providing high-volume ranibizumab services have a responsibility to audit and report adverse events (as and when they occur) with a view to establishing an evidence base that will help the community to understand the true estimate of any treatment-related adverse events.

\section{Selective versus non-selective VEGF inhibition}

The superior trial results for ranibizumab have made it the drug of choice for neovascular AMD. At this time, the role 
of selective inhibition of the $\mathrm{VEGF}_{165}$ isomer remains more limited, unless the safety concerns associated with long-term pan-VEGF blockade are confirmed. However, in clinical practice, it may be useful to consider pegatanib sodium as a second-line agent that could be used in patients with recent thromboembolic phenomena. In this vein, it may be advisable to use pegatanib sodium as a maintenance therapy in patients requiring prolonged treatment (Friberg et al 2007). As the maximum gain in vision recorded with ranibizumab treatment was obtained with the first four treatments in the MARINA and ANCHOR studies (Brown et al 2006; Rosenfeld et al 2006), it may also be prudent to consider the use of pegaptanib sodium as a secondary agent in order to maintain the effect of therapy. A recent study involving 20 patients, with all $\mathrm{CNV}$ subtypes, who were treated with bevacizumab induction followed by pegaptanib sodium maintenance therapy, demonstrated an improvement in clinical outcomes and acceptable safety profiles using the combined selective- and non-selective VEGF regimen (Hughes and Sang 2006). This hypothesis is being examined in the on-going evaLuation of Efficacy and safety in maintaining Visual acuity with sEquential treatment of neovascuLar AMD (LEVEL) study (Friberg et al 2007).

\section{Clinical implications}

Due to the nature of the existing evidence, there remains uncertainty over the relative benefits of pegaptanib sodium compared with ranibizumab and bevacizumab. Head-to-head RCTs and economic evaluations comparing the treatment alternatives are needed.

Based on the pivotal trials of pegaptanib sodium and ranibizumab, pegaptanib sodium has a more limited role as first-line therapy for neovascular AMD. However, exploratory analysis of the VISION study demonstrates better treatment response with pegaptanib sodium in early lesions (Gonzales and VISION Trial Group 2005). Similarly, reallife, clinical experience with pegaptanib sodium shows greater effectiveness than the results of the VISION study may suggest (Quiram et al 2007). It may also be useful to further investigate the effect of pegaptanib sodium in the patient group who responded best to treatment in the ranibizumab MARINA trial - those patients with small occult lesions with recent disease progression (Quiram et al 2007).

The different mechanisms of action of PDT with verteporfin and anti-angiogenic agents such as VEGF inhibitors offers the potential for synergistic effects with combination therapy. Verteporfin targets the present vascular component, whereas anti-angiogenic agents target key mediators of the angiogenic cascade. In addition, anti-angiogenic agents might counteract up-regulation of angiogenic factors (including VEGF) that occur after PDT with verteporfin (Schmidt-Erfurth et al 2003). Results from preclinical and clinical studies of combining pegaptanib sodium or ranibizumab with PDT with verteporfin warrant continued investigation (Brown et al 2006; Dhalla et al 2006; Liggett et al 2006; Heier et al 2006; Lazic et al 2007). The 1-year results of the FOCUS study demonstrate that combining ranibizumab with $\mathrm{PDT}$ with verteporfin is more effective than treatment with PDT with verteporfin alone (Heier et al 2006). Such combinations may reduce the need for repeated treatments.

\section{Cost-effectiveness}

Cost-effectiveness is another criterion against which treatment choice in neovascular AMD is assessed. However, based on the trial data currently available, it is difficult to estimate the true pharmacoeconomic benefit one agent offers over another. For example, the cost-effectiveness data of pegaptanib sodium are extrapolated from data obtained in the VISION study, but real-life clinical experience indicates that treatment with pegaptanib sodium may offer better clinical efficacy than the outcomes of the VISION study might suggest. Moreover, the number of injections each patient requires is dependent on clinical judgment and patient choice, both subjective factors that may show wide variation due to the absence of strict discontinuation criteria. Many medical retina specialists follow the re-treatment criteria used in the Prospective OCT imaging of patients with Neovascular AMD Treated with intraOcular ranibizumab (PrONTO) study (Lalwani et al 2007). However, OCT may fail to identify lesions with central atrophy and fibrosis, which can also be useful indicators of discontinuation (personal experience).

A recent study expressed cost-effectiveness as the incremental cost (IC) per vision-year saved and IC per qualityadjusted life year (QALY). When treatment was targeted to patients with a VA of $6 / 12$ to $6 / 95$ and discontinued after 2 years, or earlier if the VA fell below $6 / 95$ or by $\geq 6$ lines, the IC/QALY was estimated at $£ 8,023$ (upper $95 \% \mathrm{CI}$ $£ 20,641$ ) (Wolowacz et al 2007). The study also concluded that pegaptanib sodium was more cost-effective in patients $<75$ years of age and with better baseline VA (range 6/12 to 6/24) (Wolowacz et al 2007). Value-based medicine analysis of clinical trials that evaluated the interventions of laser photocoagulation, ITV pegaptanib sodium, and PDT with verteporfin for the treatment of classic, subfoveal CNV show that laser photocoagulation confers an improvement in quality of 
life of $4.4 \%$ compared with $5.9 \%$ with pegaptanib sodium, and an improvement of $8.1 \%$ with PDT (Brown et al 2007a). Preliminary data suggest the value gain for the treatment of occult/minimally classic CNV with ranibizumab is greater than 15\% (Brown et al 2007b). These interventions provide much better value than many routinely used medicines such as statins for the treatment of hyperlipidemia, which offer an average quality of life gain of 3.9\% (Brown et al 2007b). The economic burden of AMD on the National Health Service as a single entity has not been rigorously assessed and quantified, and analysis thus far has been based on reported blind registration and prevalence studies. Moreover, a significant number of patients with neovascular AMD may remain mis- or undiagnosed.

\section{Conclusions}

There is now a selection of agents for the treatment of neovascular AMD on offer, and treatment choice should take into consideration clinical rationale (including the safety profile of the available therapies) and cost-effectiveness. The role of pegaptanib sodium may be that of a maintenance agent, after induction with a pan-VEGF inhibitor, especially in patients who have been subject to recent MI and stroke. Only clinical trials focused in these areas, combined with analysis of post-marketing surveillance of the new anti-angiogenic agents, will help further define the optimal indications for the selective $\mathrm{VEGF}_{165}$ inhibitor.

In the light of the changing treatment paradigm brought about by the introduction of anti-angiogenic agents in neovascular AMD, many ophthalmology departments now offer fast-track AMD diagnostic services. These services not only facilitate efficient administration of the new therapies, but also aim to provide better education of the public, optometrists and ophthalmologists. Better education, fuelled by significant media interest in the area, may combine to help improve diagnosis and minimize the impact of the condition on the healthcare service.

\section{Disclosures}

The author has received travel grants and speaker fees from Pfizer.

\section{References}

Adamis AP, Altaweel M, Bressler NM, et al; Macugen Diabetic Retinopathy Study Group. 2006. Changes in retinal neovascularization after pegaptanib (Macugen) therapy in diabetic individuals. Ophthalmology, 113:23-8.

Aiello LP, Avery RL, Arrigg PG, et al. 1994. Vascular endothelial growth factor in ocular fluid of patients with diabetic retinopathy and other retinal disorders. $N$ Engl J Med, 331:1480-7.
Ambati J, Ambati BK, Yoo SH, et al. 2003. Age-related macular degeneration: etiology, pathogenesis, and therapeutic strategies. Surv Ophthalmol, 48:257-93.

Baffi J, Byrnes G, Chan CC, et al. 2000. Choroidal neovascularization in the rat induced by adenovirus mediated expression of vascular endothelial growth factor. Invest Ophthalmol Vis Sci, 41:3582-9.

Bakri SJ, Snyder MR, Reid JM, et al. 2007. Pharmacokinetics of intravitreal ranibizumab (lucentis). Ophthalmology, 114:2179-82.

Bhisitkul RB. 2006. Vascular endothelial growth factor biology: clinical implications for ocular treatments. Br J Ophthalmol, 90:1542-7.

Bressler NM, Bressler SB, Congdon NG, et al; Age-Related Eye Disease Study Research Group. 2003. Potential public health impact of Age-Related Eye Disease Study results: AREDS report no. 11. Arch Ophthalmol, 121:1621-4.

Bressler NM, Arnold J, Benchaboune M, et al; Treatment of Age-Related Macular Degeneration with Photodynamic Therapy (TAP) Study Group. 2002. Verteporfin therapy of subfoveal choroidal neovascularization in patients with age-related macular degeneration: additional information regarding baseline lesion composition's impact on vision outcomes-TAP report No. 3. Arch Ophthalmol, 120:1443-54.

Bressler NM, Bressler SB. 2000. Photodynamic therapy with verteporfin (Visudyne): impact on ophthalmology and visual sciences. Invest Ophthalmol Vis Sci, 41:624-8.

Brown DM, Kaiser PK, Michels M, et al; ANCHOR Study Group. 2006. Ranibizumab versus verteporfin for neovascular age-related macular degeneration. N Engl J Med, 355:1432-44.

Brown GC, Brown MM, Brown HC, et al. 2007a. A value-based medicine comparison of interventions for subfoveal neovascular macular degeneration. Ophthalmology, 114:1170-8.

Brown MM, Brown GC, Brown H. 2007b. Value-based medicine and interventions for macular degeneration. Curr Opin Ophthalmol, 18:194-200.

Campochiaro PA. 2007. Targeted pharmacotherapy of retinal diseases with ranibizumab. Drugs Today (Barc), 43:529-37.

Chen J, Smith LE. 2007. Retinopathy of prematurity. Angiogenesis, 10:133-40.

Cleary CA, Jungkim S, Ravikumar K, et al. 2007. Intravitreal bevacizumab in the treatment of neovascular age-related macular degeneration, 6- and 9-month results. Eye, Sep 21 (E-pub ahead of print).

Dhalla MS, Shah GK, Blinder KJ, et al. 2006. Combined photodynamic therapy with verteporfin and intravitreal bevacizumab for choroidal neovascularization in age-related macular degeneration. Retina, 26:988-93.

Dorrell M, Uusitalo-Jarvinen H, Aguilar E, et al. 2007. Ocular neovascularization: basic mechanisms and therapeutic advances. Surv Ophthalmol, 52(Suppl 1):S3-19.

Emerson MV, Lauer AK, Flaxel CJ, et al. 2007. Intravitreal bevacizumab (Avastin) treatment of neovascular age-related macular degeneration. Retina, 27:439-44.

Evans J. 1995. Causes of blindness and partial sight in England and Wales, 1990-1991. London: Her Majesty's Stationery Office.

Eyetech Study Group. 2003. Anti-vascular endothelial growth factor therapy for subfoveal choroidal neovascularization secondary to age-related macular degeneration: phase II study results. Ophthalmology, 110:979-86.

Eyetech Study Group. 2002. Preclinical and phase 1A clinical evaluation of an anti-VEGF pegylated aptamer (EYE001) for the treatment of exudative age-related macular degeneration. Retina, 22:143-52.

Ferrara N. 2004. Vascular endothelial growth factor: basic science and clinical progress. Endocr Rev, 25:581-611.

Ferris FL 3rd, Fine SL, Hyman L. 1984. Age-related macular degeneration and blindness due to neovascular maculopathy. Arch Ophthalmol, 102:1640-2.

Frank RN, Amin RH, Eliott D, et al. 1996. Basic fibroblast growth factor and vascular endothelial growth factor are present in epiretinal and choroidal neovascular membranes. Am J Ophthalmol, 122:393-403.

Friberg TR; LEVEL Study Group. 2007. Evaluation of efficacy and safety in maintaining visual acuity with sequential treatment of neovascular AMD: The LEVEL study. Invest Ophthalmol Vis Sci, 48 [ARVO e-abstract] nr. 4568. 
Gaudreault J, Fei D, Rusit J, et al. 2005. Preclinical pharmacokinetics of Ranibizumab (rhuFabV2) after a single intravitreal administration. Invest Ophthalmol Vis Sci, 46:726-33.

Genentech. 2007. Letter to healthcare providers providing latest data from the SAILOR trial. January 24.

Gonzales CR; VEGF Inhibition Study in Ocular Neovascularization (VISION) Clinical Trial Group. 2005. Enhanced efficacy associated with early treatment of neovascular age-related macular degeneration with pegaptanib sodium: an exploratory analysis. Retina, 25:815-27.

Gragoudas ES, Adamis AP, Cunningham ET Jr, et al; VEGF Inhibition Study in Ocular Neovascularization Clinical Trial Group. 2004. Pegaptanib for neovascular age-related macular degeneration. $N$ Engl $J$ Med, 351:2805-16.

Heier JS, Boyer DS, Ciulla TA, et al; FOCUS Study Group. 2006. Ranibizumab combined with verteporfin photodynamic therapy in neovascular age-related macular degeneration: year 1 results of the FOCUS Study. Arch Ophthalmol, 124:1532-42.

Houck KA, Ferrara N, Winer J, et al. 1991. The vascular endothelial growth factor family: identification of a fourth molecular species and characterization of alternative splicing of RNA. Mol Endocrinol, 5:1806-14.

Hughes MS, Sang DN. 2006. Safety and efficacy of intravitreal bevacizumab followed by pegaptanib maintenance as a treatment regimen for agerelated macular degeneration. Ophth Surg Laser Imag, 37:448-54.

Infanger M, Faramarzi S, Grosse J, et al. 2007. Expression of vascular endothelial growth factor and receptor tyrosine kinases in cardiac ischemia/reperfusion injury. Cardiovasc Pathol, 16:291-9.

Klein R, Klein BE, Linton KL. 1992. Prevalence of age-related maculopathy. The Beaver Dam Eye Study. Ophthalmolology, 99:933-43.

Krzystolik MG, Afshari MA, Adamis AP, et al. 2002. Prevention of experimental choroidal neovascularization with intravitreal antivascular endothelial growth factor antibody fragment. Arch Ophthalmol, 120:338-46.

Larsson A, Sköldenberg E, Ericson H. 2002. Serum and plasma levels of FGF-2 and VEGF in healthy blood donors. Angiogenesis, 5:107-10.

Lazic R, Gabric N. 2007. Verteporfin therapy and intravitreal bevacizumab combined and alone in choroidal neovascularization due to age-related macular degeneration. Ophthalmology, 114:1179-85.

Liew G, Mitchell P, Gillies MC, et al. 2007. Ranibizumab for neovascular age-related macular degeneration. $N$ Engl J Med, 356:747-50.

Liggett PE, Colina J, Chaudhry NA, et al. 2006. Triple therapy of intravitreal triamcinolone, photodynamic therapy, and pegaptanib sodium for choroidal neovascularization. Am J Ophthalmol, 142:1072-4.

Lopez PF, Sippy BD, Lambert HM, et al. 1996. Transdifferentiated retinal pigment epithelial cells are immunoreactive for vascular endothelial growth factor in surgically excised age-related macular degenerationrelated choroidal neovascular membranes. Invest Ophthalmol Vis Sci, 37:855-68.

Macugen AMD Study Group; Apte RS, Modi M, Masonson H, et al. 2007. Pegaptanib 1-year systemic safety results from a safety-pharmacokinetic trial in patients with neovascular age-related macular degeneration. Ophthalmology, 114:1702-12.

Macular Photocoagulation Study Group. 1993. Laser photocoagulation of subfoveal neovascular lesions of age-related macular degeneration Updated findings from two clinical trials, Arch Ophthalmol, 111:1200-9.

National Institute for Health and Clinical Excellence (NICE). 2003. Guidance on the use of photodynamic therapy for age-related macular degeneration. Technology appraisal 68. London.

$\mathrm{Ng}$ EW, Adamis AP. 2005. Targeting angiogenesis, the underlying disorder in neovascular age-related macular degeneration. Can J Ophthalmol, 40:352-68.
Owen CG, Fletcher AE, Donoghue M, et al. 2003. How big is the burden of visual loss caused by age related macular degeneration in the United Kingdom? Br J Ophthalmol, 87:312-7.

Pe'er J, Folberg R, Itin A, et al. 1998. Vascular endothelial growth factor upregulation in human central retinal vein occlusion. Ophthalmology, 105:412-6.

Pertovaara L, Kaipainen A, Mustonen T, et al. 1994. Vascular endothelial growth factor is induced in response to transforming growth factor- $\beta$ in fibroblastic and epithelial cells. J Biol Chem, 269:6271-4.

Pierce EA, Foley ED, Smith LE. 1996. Regulation of vascular endothelial growth factor by oxygen in a model of retinopathy of prematurity. Arch Ophthalmol, 114:1219-28.

Quiram PA, Hassan TS, Williams GA. 2007. Treatment of naive lesions in neovascular age-related macular degeneration with pegaptanib. Retina, 27:851-6.

Rosenfeld PJ, Brown DM, Heier JS, et al; MARINA Study Group. 2006. Ranibizumab for neovascular age-related macular degeneration. $N$ Engl $J$ Med, 355:1419-31.

Ruckman J, Green LS, Beeson J, et al. 1998. 2'-Fluoropyrimidine RNAbased aptamers to the 165-amino acid form of vascular endothelial growth factor (VEGF165). Inhibition of receptor binding and VEGFinduced vascular permeability through interactions requiring the exon 7-encoded domain. J Biol Chem, 273:20556-67.

Schmidt-Erfurth U, Schlotzer-Schrehard U, Cursiefen C, et al. 2003. Influence of photodynamic therapy on expression of vascular endothelial growth factor (VEGF), VEGF receptor 3, and pigment epitheliumderived factor. Invest Ophthalmol Vis Sci, 44:4473-80.

Steinbrook R. 2006. The price of sight - ranibizumab, bevacizumab, and the treatment of macular degeneration. $N$ Engl J Med, 355:1409-12.

Stephan CC, Brock TA. 1996. Vascular endothelial growth factor, a multifunctional polypeptide. $P$ R Health Sci J, 15:169-78.

Tolentino MJ, Miller JW, Gragoudas ES, et al. 1996. Intravitreous injections of vascular endothelial growth factor produce retinal ischemia and microangiopathy in an adult primate. Ophthalmology, 103:1820-8.

Treatment of Age-Related Macular Degeneration with Photodynamic Therapy (TAP) Study Group. 1999. Photodynamic therapy of subfoveal choroidal neovascularization in age-related macular degeneration with verteporfin One-year results of 2 randomized clinical trials - TAP report. Arch Ophthalmol, 117:1329-45.

Tripathi RC, Li J, Tripathi BJ, et al. 1998. Increased level of vascular endothelial growth factor in aqueous humor of patients with neovascular glaucoma. Ophthalmology, 105:232-7.

Usui T, Ishida S, Yamashiro K, et al. 2004. VEGF164(165) as the pathological isoform: differential leukocyte and endothelial responses through VEGFR1 and VEGFR2. Invest Ophthalmol Vis Sci, 45:368-74.

Waheed NK, Miller JW. 2004. Aptamers, intramers, and vascular endothelial growth factor. Int Ophthalmol Clin, 44:11-22.

Wolowacz SE, Roskell N, Kelly S, et al. 2007. Cost effectiveness of pegaptanib for the treatment of age-related macular degeneration in the UK. Pharmacoeconomics, 25:863-79.

Wong TY, Liew G, Mitchell P. 2007. Clinical update: new treatments for age-related macular degeneration. Lancet, 370:204-6.

Wu L, Martinez-Castellanos MA, Quiroz-Mercado H, et al; for the Pan American Collaborative Retina Group (PACORES). 2008. Twelvemonth safety of intravitreal injections of bevacizumab (Avastin(R)): results of the Pan-American Collaborative Retina Study Group (PACORES). Graefes Arch Clin Exp Ophthalmol, 246:81-7.

Zlokovic BV. 2006. Remodeling after stroke. Nat Med, 12:390-1. 